\title{
Assessing the quality of sardine based on biogenic amines using a fuzzy logic model
}

\begin{abstract}
There is an increasing concern about the quality and quality assessment procedures of seafood. In the present study, a model to assess fish quality based on biogenic amine contents using fuzzy logic model (FLM) is proposed. The fish used was sardine (Sardinella sp.) where the production of eight biogenic amines was monitored over fifteen days of storage at 0,3 and $10^{\circ} \mathrm{C}$. Based on the results, histamine, putrescine and cadaverine were selected as input variables and twelve quality grades were considered for quality of fish as output variables for the FLM. Input data were processed by rules established in the model and were then defuzzified according to defined output variables. Finally, the quality of fish was evaluated using the designed model and Pearson correlation between storage times with quality of fish showed $\mathrm{r}=0.97,0.95$ and 1 for fish stored at 0,3 and $10^{\circ} \mathrm{C}$, respectively.
\end{abstract}

Keyword: Biogenic amines; Cadaverine; Fish quality; Fuzzy logic model; Histamine; Putrescine 